\title{
Die ganze Breite des Faches
}

\section{The Whole Spectrum of the Specialty}

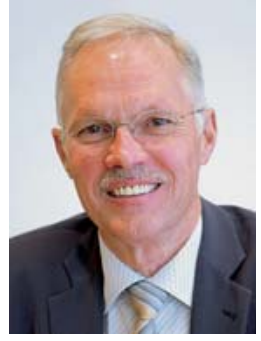

F. U. Niethard

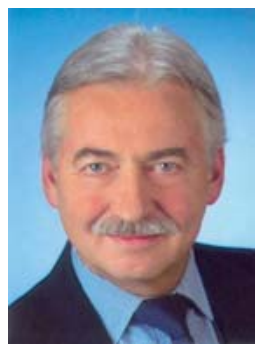

K. Weise
Die neue Zeitschrift für Orthopädie und Unfallchirurgie besteht nun zwei Jahre. Herausgeber und Rubrik-Herausgeber, Autoren und Leser der Zeitschrift werden sorgfältig beobachtet haben, welche Veränderungen sich vollzogen haben [1]. Für die Herausgeber sind diese durchweg positiv: Die Anzahl der eingehenden Manuskripte ist gestiegen. Dementsprechend können noch mehr qualitativ hochwertige Manuskripte publiziert werden.

Dies wird sich im Verlaufe der nächsten Jahre positiv auf den Impact-Faktor auswirken. Für 2008 und 2009 wird es einen kombinierten ImpactFaktor geben, der sich auf beide Zeitschriftentitel bezieht, die in den beiden relevanten Jahren zitiert wurden.

Besonders erfreulich ist, dass sich bereits innerhalb der kurzen Zeit ein ausgewogenes Verhältnis zwischen originär orthopädischen und originär unfallchirurgischen Arbeiten eingestellt hat. Eine strikte Trennung ist bei vielen Themen ohnehin nicht möglich. So ist z.B. der Riss der Rotatorenmanschette sowohl ein orthopädisches als auch unfallchirurgisches Thema: So gesehen weisen zahlreiche Manuskripte eine orthopädisch-unfallchirurgische Thematik auf und diese inhaltlichen Überschneidungen waren ja auch der Grund für die neue Weiterbildungsordnung zum Arzt für Orthopädie und Unfallchirurgie und letztendlich für den Zusammenschluss der Gesellschaften [2].

Die Herausgeber sind also erfreut, dass sie in jedem Heft einen bunten Strauß von Publikationen aus dem gesamten Bereich von Orthopädie und Unfallchirurgie anbieten können, der die volle Breite des Faches widerspiegelt. Auch in diesem Heft findet sich von der Kinderorthopädie über die Schmerztherapie, die Unfallchirurgie und die Knieendoprothetik bis zur Grundlagenforschung ein großes Angebot von Originalarbeiten, die die Leistungsfähigkeit des Faches Orthopädie und Unfallchirurgie abbilden. In vielen Fällen repräsentieren die Titelüberschriften Substrukturen des neuen Faches, die sich nun auch im Gesamtvorstand der Deutschen Gesellschaft für Orthopädie und Unfallchirurgie (DGOU) wiederfinden. Im Vereinsverband der DGOU sind nämlich auch zahlreiche Sektionen vertreten, die für die zunehmende Spezialisierung des Faches stehen. Die Herausgeber sehen es als eine wichtige Aufgabe an, die Sektionen in die Arbeit der Zeitschrift für Orthopädie und Unfallchirurgie einzubinden. Die Leiter der Sektionen sind Kraft ihres Amtes Mitglied im Beirat. Die Zeitschrift für Orthopädie und Unfallchirurgie begleitet die DGOU und ihre Sektionen publizistisch und ist Forum für ihre Ergebnisse und Arbeiten. So ist die Zeitschrift für Orthopädie und Unfallchirurgie für jeden interessant, der sich über die aktuellen Entwicklungen dieser Fachgebiete orientieren will. Ein Angebot nicht nur für den chirurgisch, sondern auch konservativ tätigen Kollegen, wie die in diesem Heft veröffentlichten Beiträge zur Schmerztherapie zeigen [3-5].

Dieses Heft zeigt auch, dass sich die Zeitschrift für Orthopädie und Unfallchirurgie den aktuellen Themen stellt und diese mit eigenen Beiträgen bereichern kann. Die Diskussion über iatrogene Schäden spielt derzeit in der Öffentlichkeit eine bedeutende Rolle. Der einleitende Artikel aus der Kinderorthopädie über Fehler in der Diagnose und Behandlung der Epiphysiolysis capitis femoris [6] belegt, dass die Zeitschrift durch gute Publikationen zur Fehleranalyse mehr zur „Fehlerkultur" beiträgt als mancher journalistische Schnellschuss. „Aus Fehlern lernen“ heißt, dass man zunächst darüber berichtet und spricht und zwar auf wissenschaftlichem Niveau [7]. Dies geschieht in dieser Zeitschrift.

\section{F. U. Niethard, Aachen \\ K. Weise, Tübingen}

\section{Literatur}

1 Weise K, Niethard FU. Die Entwicklung der Zeitschrift für Orthopädie und Unfallchirurgie - aus Sicht der Herausgeber. Z Orthop Unfall 2008; 146: 311

2 Niethard FU, Weise K. Ein historischer Tag - Deutsche Gesellschaft für Orthopädie und Unfallchirurgie gegründet. Z Orthop Unfall 2008; 146: 437-438

3 Machacek $P$, Friedrich $M$, Hahne J. Angaben über Schmerzen im Bereich des Bewegungsapparates: eine repräsentative Befragung der österreichischen Bevölkerung. Z Orthop Unfall 2008; 146: 730-735

4 Andresen R, Rathmer S, Nickel J et al. Ambulante CTgestützte thorakale Sympathikus-Blockade als zusätzliche Therapieoption bei komplexem regionalen Schmerzsyndrom Typ I nach Sportverletzungen. Z Orthop Unfall 2008; 146: 736-741

5 Riesner HJ, Zeidler C, Schreiber $H$ et al. Eine additive Therapie chronischer Schmerzen bei fortgeschrittener Gon-/Coxarthrose mit dem selektiven Serotoninre-up-take-Hemmer Fluvoxamin $\left(\right.$ Fevarin $\left.^{\circledR}\right)$. Z Orthop Unfall 2008; 146: 742-746

6 Püschmann H, Vinz H, Neu J. Fehler in der Diagnose und Behandlung der Epiphysiolysis capitis femoris. Z Orthop Unfall 2008; 146: 710-714

7 Niethard FU, Weise K. Iatrogene Schäden - Fehlervermeidung in Orthopädie und Unfallchirurgie. Z Orthop Unfall 2008; 146: 173-174

Schnarrenbergstraße 95 72076 Tübingen

Tel.: 07071/606-1001

Fax: 07071/606-1002

weise@bgu-tuebingen.de 